\title{
EXPLICIT EVALUATION OF CERTAIN SUMS OF MULTIPLE ZETA-STAR VALUES
}

\author{
SHUJI YAMAMOTO
}

\begin{abstract}
Bowman and Bradley proved an explicit formula for the sum of multiple zeta values whose indices are the sequence $(3,1,3,1, \ldots, 3,1)$ with a number of 2's inserted. Kondo, Saito and Tanaka considered the similar sum of multiple zeta-star values and showed that this value is a rational multiple of a power of $\pi$. In this paper, we give an explicit formula for the rational part. In addition, we interpret the result as an identity in the harmonic algebra.
\end{abstract}

\section{INTRODUCTION}

Let us consider the multiple zeta values (MZV, for short)

$$
\zeta\left(k_{1}, \ldots, k_{n}\right)=\sum_{m_{1}>\cdots>m_{n}>0} \frac{1}{m_{1}^{k_{1}} \cdots m_{n}^{k_{n}}} .
$$

In some cases, explicit evaluations are known for these values or sums of them. For example, there are the formulas

$$
\begin{aligned}
\zeta\left(\{2\}^{q}\right) & =\frac{\pi^{2 q}}{(2 q+1) !} \\
\zeta\left(\{3,1\}^{p}\right) & =\frac{\pi^{4 p}}{(2 p+1)(4 p+1) !}
\end{aligned}
$$

(the notation \{\}$^{p}$ means that the sequence in the bracket is repeated $p$-times). In fact, these values are the special cases $s(0, q)$ and $s(p, 0)$ of the following sums of MZVs

$$
s(p, q)=\sum_{\substack{j_{0}, j_{1}, \ldots, j_{2 p} \geq 0 \\ j_{0}+j_{1}+\cdots+j_{2 p}=q}} \zeta\left(\{2\}^{j_{0}}, 3,\{2\}^{j_{1}}, 1,\{2\}^{j_{2}}, 3, \ldots, 3,\{2\}^{j_{2 p-1}}, 1,\{2\}^{j_{2 p}}\right),
$$

for which an explicit formula was given by Bowman-Bradley [BB]:

$$
s(p, q)=\left(\begin{array}{c}
2 p+q \\
q
\end{array}\right) \frac{\pi^{4 p+2 q}}{(2 p+1)(4 p+2 q+1) !} .
$$

On the other hand, we may also consider the multiple zeta-star values (MZSV for short)

$$
\zeta^{\star}\left(k_{1}, \ldots, k_{n}\right)=\sum_{m_{1} \geq \cdots \geq m_{n} \geq 1} \frac{1}{m_{1}^{k_{1}} \cdots m_{n}^{k_{n}}} .
$$

2010 Mathematics Subject Classification. Primary 11M32, Secondary 05A15.

Key words and phrases. multiple zeta values, multiple zeta-star values, harmonic algebra, Bowman-Bradley theorem, Kondo-Saito-Tanaka theorem.

This work was supported by Grant-in-Aid for JSPS Fellows 21.5093. 
As an analogue of $s(p, q)$, we put

$$
s^{\star}(p, q)=\sum_{\substack{j_{0}, j_{1}, \ldots, j_{2 p} \geq 0 \\ j_{0}+j_{1}+\cdots+j_{2 p}=q}} \zeta^{\star}\left(\{2\}^{j_{0}}, 3,\{2\}^{j_{1}}, 1,\{2\}^{j_{2}}, 3, \ldots, 3,\{2\}^{j_{2 p-1}}, 1,\{2\}^{j_{2 p}}\right) .
$$

Then the theorem of Kondo-Saito-Tanaka KST states that $s^{\star}(p, q) \in \mathbb{Q} \pi^{4 p+2 q}$ (see also [T]). The rational part, however, has not been given explicitly except for the cases $p=0$ (Zlobin $[\mathrm{Z}]$ ) and $q=0,1$ (Muneta $[\mathbf{M}]$ ). The formula for $p=0$ is

$$
s^{\star}(0, q)=\zeta^{\star}\left(\{2\}^{q}\right)=\left(2^{2 q}-2\right) \frac{(-1)^{q-1} B_{2 q}}{(2 q) !} \pi^{2 q}
$$

( $B_{2 q}$ is the $2 q$-th Bernoulli number).

In this paper, we prove the following relation between $s(p, q)$ and $s^{\star}(p, q)$ :

Theorem 1.1. For any $p, q \geq 0$, we have

$$
s^{\star}(p, q)=\sum_{\substack{2 i+k+u=2 p \\
j+l+v=q}}(-1)^{j+k}\left(\begin{array}{c}
k+l \\
k
\end{array}\right)\left(\begin{array}{c}
u+v \\
u
\end{array}\right) s(i, j) \zeta^{\star}\left(\{2\}^{k+l}\right) \zeta^{\star}\left(\{2\}^{u+v}\right) .
$$

By substituting (1.3) and (1.4) into (1.5), we obtain an explicit formula for the value of $s^{\star}(p, q)$ :

$$
\frac{s^{\star}(p, q)}{\pi^{4 p+2 q}}=\sum_{\substack{2 i+k+u=2 p \\
j+l+v=q}}(-1)^{j+k}\left(\begin{array}{c}
k+l \\
k
\end{array}\right)\left(\begin{array}{c}
u+v \\
u
\end{array}\right)\left(\begin{array}{c}
2 i+j \\
j
\end{array}\right) \frac{\beta_{k+l} \beta_{u+v}}{(2 i+1)(4 i+2 j+1) !},
$$

where

$$
\beta_{r}=\left(2^{2 r}-2\right) \frac{(-1)^{r-1} B_{2 r}}{(2 r) !} .
$$

In particular, when $q=0$, we can reproduce Muneta's expression for $s^{\star}(p, 0)[\mathrm{M}$, Theorem B]. When $q=1$, however, our result appears different from his formula for $s^{\star}(p, 1)[\mathrm{M}$, Theorem C].

In fact, our result is slightly more general than Theorem 1.1. namely, the numbers $3,1,2$ are replaced by arbitrary positive integers $a, b, c$ such that $a+b=2 c$ and $a \geq 2$. Moreover, it is shown as a corollary of the corresponding identity between finite partial sums of multiple zeta series (see Theorem 2.11). In $\S 3$, we also give an interpretation as an identity in the harmonic algebra.

\section{Generating SERIES OF TRUNCATED SUMS}

For an integer $m \geq 0$ and an index $\mathbf{k}=\left(k_{1}, \ldots, k_{n}\right)\left(k_{1}, \ldots, k_{n} \geq 1\right)$, we define finite sums $\zeta_{m}(\mathbf{k})$ and $\zeta_{m}^{\star}(\mathbf{k})$ by truncating the series for $\zeta(\mathbf{k})$ and $\zeta^{\star}(\mathbf{k})$, respectively:

$$
\zeta_{m}(\mathbf{k})=\sum_{m \geq m_{1}>\cdots>m_{n}>0} \frac{1}{m_{1}^{k_{1}} \cdots m_{n}^{k_{n}}}, \quad \zeta_{m}^{\star}(\mathbf{k})=\sum_{m \geq m_{1} \geq \cdots \geq m_{n} \geq 1} \frac{1}{m_{1}^{k_{1}} \cdots m_{n}^{k_{n}}} .
$$

Here an empty sum is read as 0 . When $n=0$, we denote by $\varnothing$ the unique index of length zero, and put $\zeta_{m}(\varnothing)=\zeta_{m}^{\star}(\varnothing)=1$ for all $m \geq 0$. 
In the following, we fix positive integers $a, b$ and $c$ satisfying $a+b=2 c$. For integers $p, q \geq 0$, let $I_{p, q}=I_{p, q}^{a, b, c}$ denote the set of all indices obtained by shuffling two sequences $\left(\{a, b\}^{q}\right)$ and $\left(\{c\}^{p}\right)$. For example,

$$
\begin{gathered}
I_{0,0}=\{\varnothing\}, \quad I_{1,1}=\{(a, b, c),(a, c, b),(c, a, b)\}, \\
I_{1,2}=\{(a, b, c, c),(a, c, b, c),(a, c, c, b),(c, a, b, c),(c, a, c, b),(c, c, a, b)\} .
\end{gathered}
$$

Let us consider the sums of truncated MZVs and MZSVs analogous to $s(p, q)$ and $s^{\star}(p, q)$ in the introduction:

$$
s_{m}(p, q)=\sum_{\mathbf{k} \in I_{p, q}} \zeta_{m}(\mathbf{k}), \quad s_{m}^{\star}(p, q)=\sum_{\mathbf{k} \in I_{p, q}} \zeta_{m}^{\star}(\mathbf{k}) .
$$

Then Theorem 1.1 is obtained from the following identity by putting $(a, b, c)=$ $(3,1,2)$ and letting $m \rightarrow \infty$ :

Theorem 2.1. For any $p, q \geq 0$ and $m \geq 0$, we have

$$
s_{m}^{\star}(p, q)=\sum_{\substack{2 i+k+u=2 p \\
j+l+v=q}}(-1)^{j+k}\left(\begin{array}{c}
k+l \\
k
\end{array}\right)\left(\begin{array}{c}
u+v \\
u
\end{array}\right) s_{m}(i, j) \zeta_{m}^{\star}\left(\{c\}^{k+l}\right) \zeta_{m}^{\star}\left(\{c\}^{u+v}\right) .
$$

If we put

$$
\begin{aligned}
& F_{m}(x, y)=\sum_{p, q \geq 0} s_{m}(p, q) x^{2 p} y^{q}, \quad H_{m}(z)=\sum_{r \geq 0} \zeta_{m}\left(\{c\}^{r}\right) z^{r}, \\
& F_{m}^{\star}(x, y)=\sum_{p, q \geq 0} s_{m}^{\star}(p, q) x^{2 p} y^{q}, \quad H_{m}^{\star}(z)=\sum_{r \geq 0} \zeta_{m}^{\star}\left(\{c\}^{r}\right) z^{r},
\end{aligned}
$$

then it is not difficult to see that Theorem 2.1 is equivalent to the following generating series identity:

\section{Theorem 2.2.}

$$
F_{m}^{\star}(x, y)=F_{m}(x,-y) H_{m}^{\star}(y-x) H_{m}^{\star}(y+x) .
$$

Remark 2.3. Prof. Kaneko pointed out that, since

$$
H_{m}^{\star}(z)=\prod_{l=1}^{m}\left(1-\frac{z}{l^{c}}\right)^{-1}=H_{m}(-z)^{-1}
$$

(2.2) can be written more symmetrically as

$$
\frac{F_{m}^{\star}(x, y)}{H_{m}^{\star}(x+y)}=\frac{F_{m}(x,-y)}{H_{m}(x-y)} .
$$

To prove the identity (2.2), we introduce another kind of sums and their generating series. We define $J_{p, q}=J_{p, q}^{a, b, c}$ as the set of all shuffles of $\left(b,\{a, b\}^{q}\right)$ and $\left(\{c\}^{p}\right)$, e.g.

$$
J_{0,0}=\{(b)\}, \quad J_{1,1}=\{(b, a, b, c),(b, a, c, b),(b, c, a, b),(c, b, a, b)\},
$$

and put

$$
\begin{aligned}
t_{m}(p, q) & =\sum_{\mathbf{k} \in J_{p, q}} \zeta_{m}(\mathbf{k}), \quad G_{m}(x, y)=\sum_{p, q \geq 0} t_{m}(p, q) x^{2 p+1} y^{q}, \\
t_{m}^{\star}(p, q) & =\sum_{\mathbf{k} \in J_{p, q}} \zeta_{m}^{\star}(\mathbf{k}), \quad G_{m}^{\star}(x, y)=\sum_{p, q \geq 0} t_{m}^{\star}(p, q) x^{2 p+1} y^{q} .
\end{aligned}
$$


Lemma 2.4. For $m \geq 0$, we have

$$
\begin{aligned}
& \left(\begin{array}{l}
F_{m}(x, y) \\
G_{m}(x, y)
\end{array}\right)=U_{m} U_{m-1} \cdots U_{1}\left(\begin{array}{l}
1 \\
0
\end{array}\right), \\
& \left(\begin{array}{c}
F_{m}^{\star}(x, y) \\
G_{m}^{\star}(x, y)
\end{array}\right)=V_{m} V_{m-1} \cdots V_{1}\left(\begin{array}{l}
1 \\
0
\end{array}\right),
\end{aligned}
$$

where

$$
\begin{aligned}
U_{l} & =\left(\begin{array}{cc}
1+\frac{y}{l^{c}} & \frac{x}{l^{a}} \\
\frac{x}{l^{b}} & 1+\frac{y}{l^{c}}
\end{array}\right), \\
V_{l} & =\frac{1}{\left(1-\frac{y-x}{l^{c}}\right)\left(1-\frac{y+x}{l^{c}}\right)}\left(\begin{array}{cc}
1-\frac{y}{l^{c}} & \frac{x}{l^{a}} \\
\frac{x}{l^{b}} & 1-\frac{y}{l^{c}}
\end{array}\right) .
\end{aligned}
$$

Proof. For $m=0$, both (2.3) and (2.4) are obvious. For $m \geq 1$, we write

$$
\begin{aligned}
F_{m}(x, y) & =\sum_{p, q \geq 0} \sum_{\mathbf{k} \in I_{p, q}} \zeta_{m}(\mathbf{k}) x^{2 p} y^{q} \\
& =\sum_{p, q \geq 0} \sum_{\left(k_{1}, \ldots, k_{2 p+q}\right) \in I_{p, q}} \sum_{m \geq m_{1}>\cdots>m_{2 p+q} \geq 1} \frac{x^{2 p} y^{q}}{m_{1}^{k_{1}} \cdots m_{2 p+q}^{k_{2 p+q}}} .
\end{aligned}
$$

We decompose this series into three partial sums, each consisting of the terms such that (i) $m_{1}<m$, (ii) $m_{1}=m$ and $k_{1}=a$, or (iii) $m_{1}=m$ and $k_{1}=c$, respectively. Then we obtain the equality

$$
F_{m}(x, y)=F_{m-1}(x, y)+\frac{x}{m^{a}} G_{m-1}(x, y)+\frac{y}{m^{c}} F_{m-1}(x, y) .
$$

Similarly, we also have

$$
G_{m}(x, y)=G_{m-1}(x, y)+\frac{x}{m^{b}} F_{m-1}(x, y)+\frac{y}{m^{c}} G_{m-1}(x, y) .
$$

Combining them together, we get

$$
\left(\begin{array}{c}
F_{m}(x, y) \\
G_{m}(x, y)
\end{array}\right)=U_{m}\left(\begin{array}{c}
F_{m-1}(x, y) \\
G_{m-1}(x, y)
\end{array}\right)
$$

and hence (2.3) by induction.

In a similar way, we can show that

$$
\begin{aligned}
& F_{m}^{\star}(x, y)=F_{m-1}^{\star}(x, y)+\frac{x}{m^{a}} G_{m}^{\star}(x, y)+\frac{y}{m^{c}} F_{m}^{\star}(x, y), \\
& G_{m}^{\star}(x, y)=G_{m-1}^{\star}(x, y)+\frac{x}{m^{b}} F_{m}^{\star}(x, y)+\frac{y}{m^{c}} G_{m}^{\star}(x, y),
\end{aligned}
$$

that is,

$$
\left(\begin{array}{cc}
1-\frac{y}{m^{c}} & -\frac{x}{m^{a}} \\
-\frac{x}{m^{b}} & 1-\frac{y}{m^{c}}
\end{array}\right)\left(\begin{array}{l}
F_{m}(x, y) \\
G_{m}(x, y)
\end{array}\right)=\left(\begin{array}{c}
F_{m-1}(x, y) \\
G_{m-1}(x, y)
\end{array}\right)
$$

Since

$$
\left(\begin{array}{cc}
1-\frac{y}{m^{c}} & -\frac{x}{m^{a}} \\
-\frac{x}{m^{b}} & 1-\frac{y}{m^{c}}
\end{array}\right)^{-1}=V_{m}
$$

under the assumption $a+b=2 c$, we obtain (2.4) by induction. 
Now it is easy to prove Theorem 2.2. Indeed, the identities (2.3) and (2.4) imply that

$$
\begin{aligned}
\left(\begin{array}{c}
F_{m}^{\star}(x, y) \\
G_{m}^{\star}(x, y)
\end{array}\right) & =\prod_{l=1}^{m}\left\{\left(1-\frac{y-x}{l^{c}}\right)\left(1-\frac{y+x}{l^{c}}\right)\right\}^{-1} \cdot\left(\begin{array}{c}
F_{m}(x,-y) \\
G_{m}(x,-y)
\end{array}\right) \\
& =H_{m}^{\star}(y-x) H_{m}^{\star}(y+x)\left(\begin{array}{c}
F_{m}(x,-y) \\
G_{m}(x,-y)
\end{array}\right) .
\end{aligned}
$$

Remark 2.5. In the above proof, it is also shown that

$$
t_{m}^{\star}(p, q)=\sum_{\substack{2 i+k+u=2 p \\
j+l+v=q}}(-1)^{j+k}\left(\begin{array}{c}
k+l \\
k
\end{array}\right)\left(\begin{array}{c}
u+v \\
u
\end{array}\right) t_{m}(i, j) \zeta_{m}^{\star}\left(\{c\}^{k+l}\right) \zeta_{m}^{\star}\left(\{c\}^{u+v}\right) .
$$

\section{IDENTITIES IN THE HARMONIC ALGEBRA}

In this section, we give algebraic interpretations of identities (2.1) and (2.5). First we recall the setup of harmonic algebra (see [IKOO] for a more general discussion).

Let $\mathfrak{H}^{1}=\mathbb{Q}\left\langle z_{k} \mid k \geq 1\right\rangle$ be the free $\mathbb{Q}$-algebra generated by countable number of variables $z_{k}(k=1,2,3, \ldots)$. The harmonic product $*$ is the $\mathbb{Q}$-bilinear product on $\mathfrak{H}^{1}$ defined by

$$
\begin{gathered}
w * 1=1 * w=w \\
z_{k} w * z_{l} w^{\prime}=z_{k}\left(w * z_{l} w^{\prime}\right)+z_{l}\left(z_{k} w * w^{\prime}\right)+z_{k+l}\left(w * w^{\prime}\right)
\end{gathered}
$$

for $k, l \geq 1$ and $w, w^{\prime} \in \mathfrak{H}^{1}$. It is known that $\mathfrak{H}^{1}$ equipped with the product $*$ becomes a unitary commutative $\mathbb{Q}$-algebra, denoted by $\mathfrak{H}_{*}^{1}$.

For an integer $m \geq 0$, we define a $\mathbb{Q}$-linear map $Z_{m}: \mathfrak{H}^{1} \longrightarrow \mathbb{Q}$ by

$$
Z_{m}(1)=1, \quad Z_{m}\left(z_{k_{1}} \cdots z_{k_{n}}\right)=\zeta_{m}\left(k_{1}, \ldots, k_{n}\right) .
$$

In fact, $Z_{m}$ is a $\mathbb{Q}$-algebra homomorphism from $\mathfrak{H}_{*}^{1}$ to $\mathbb{Q}$. Moreover, we define a $\mathbb{Q}$-linear transformation on $\mathfrak{H}^{1}$ by

$$
S(1)=1, \quad S\left(z_{k}\right)=z_{k}, \quad S\left(z_{k} z_{l} w\right)=z_{k} S\left(z_{l} w\right)+z_{k+l} S(w)
$$

and put $Z_{m}^{\star}=Z_{m} \circ S$, so that

$$
Z_{m}^{\star}\left(z_{k_{1}} \cdots z_{k_{n}}\right)=\zeta_{m}^{\star}\left(k_{1}, \ldots, k_{n}\right)
$$

holds for any $k_{1}, \ldots, k_{n} \geq 1$.

Now let us put

$$
\mathfrak{s}_{p, q}=\sum_{\left(k_{1}, \ldots, k_{2 p+q}\right) \in I_{p, q}} z_{k_{1}} \cdots z_{k_{2 p+q}}, \quad \mathfrak{t}_{p, q}=\sum_{\left(k_{1}, \ldots, k_{2 p+q+1}\right) \in J_{p, q}} z_{k_{1}} \cdots z_{k_{2 p+q+1}} .
$$

Then the fact that the identity (2.1) holds for all $m \geq 0$ suggests that the identities

$$
\begin{aligned}
S\left(\mathfrak{s}_{p, q}\right) & =\sum_{\substack{2 i+k+u=2 p \\
j+l+v=q}}(-1)^{j+k}\left(\begin{array}{c}
k+l \\
k
\end{array}\right)\left(\begin{array}{c}
u+v \\
u
\end{array}\right) \mathfrak{s}_{i, j} * S\left(z_{c}^{k+l}\right) * S\left(z_{c}^{u+v}\right), \\
S\left(\mathfrak{t}_{p, q}\right) & =\sum_{\substack{2 i+k+u=2 p \\
j+l+v=q}}(-1)^{j+k}\left(\begin{array}{c}
k+l \\
k
\end{array}\right)\left(\begin{array}{c}
u+v \\
u
\end{array}\right) \mathfrak{t}_{i, j} * S\left(z_{c}^{k+l}\right) * S\left(z_{c}^{u+v}\right)
\end{aligned}
$$

hold in $\mathfrak{H}^{1}$. Indeed, this speculation is justified by the following theorem: 
Theorem 3.1. For $w \in \mathfrak{H}^{1}$, denote the rational sequence $\left\{Z_{m}(w)\right\}_{m \geq 0}$ by $\mathcal{Z}(w)$. Then the resulting $\mathbb{Q}$-algebra homomorphism $\mathcal{Z}: \mathfrak{H}_{*}^{1} \longrightarrow \mathbb{Q}^{\mathbb{N}}$ is injective.

If we put $\mathfrak{H}_{>0}^{1}=\bigoplus_{k \geq 1} z_{k} \mathfrak{H}^{1}$, it is obvious from the definition of $Z_{m}$ that $\mathfrak{H}_{>0}^{1}=$ $\operatorname{Ker} Z_{0}$. Hence it suffices to consider the map

$$
\mathfrak{H}_{>0}^{1} \longrightarrow \mathbb{Q}^{\mathbb{Z}_{>0}} ; w \longmapsto\left\{Z_{m}(w)\right\}_{m>0} .
$$

The injectivity of this map is an immediate consequence of the following theorem, which is obtained by specializing Corollary 5.6 in $[\mathrm{Br}$ :

Theorem 3.2. The multiple polylogarithm functions

$$
L i_{\mathbf{k}}(t)=\sum_{m_{1}>\cdots>m_{n}>0} \frac{t^{m_{1}}}{m_{1}^{k_{1}} \cdots m_{n}^{k_{n}}}=\sum_{m>0}\left(\zeta_{m}(\mathbf{k})-\zeta_{m-1}(\mathbf{k})\right) t^{m},
$$

for $\mathbf{k}=\left(k_{1}, \ldots, k_{n}\right) \in\left(\mathbb{Z}_{>0}\right)^{n}$ and $n \geq 1$, are linearly independent over the ring $\mathbb{C}[t, 1 / t, 1 /(1-t)]$.

Remark 3.3. It is also possible to prove the identities (3.1) and (3.2) directly, by making computations similar to the proof of Proposition 4 in [IKOO, in the matrix algebra $M_{2}\left(\mathfrak{H}_{*}^{1}[[x, y]]\right)$.

\section{REFERENCES}

[BB] D. Bowman and D. M. Bradley, The algebra and combinatorics of shuffles and multiple zeta values, J. Combin. Theory Ser. A 97 (2002), 43-61.

[Br] F. C. S. Brown, Multiple zeta values and periods of moduli spaces $\overline{\mathfrak{M}}_{0, n}$, Ann. Scient. Éc. Norm. Sup. 42 (2009), 371-489.

[KST] H. Kondo, S. Saito and T. Tanaka, The Bowman-Bradley theorem for multiple zeta-star values, preprint.

[IKOO] K. Ihara, J. Kajikawa, Y. Ohno, J. Okuda, Multiple zeta values vs. multiple zeta-star values, J. Alg. 332 (2011), 187-208.

[M] S. Muneta, On some explicit evaluations of multiple zeta-star values, J. Number Theory, 128 (2008), 2538-2548.

[T] T. Tanaka, A simple proof of certain formula for multiple zeta-star values, J. Alg., Number Theory: Adv. Appl. 3 (2010), 97-110.

[Z] S. A. Zlobin, Generating functions for the values of a multiple zeta function, Vestnik Moskov. Univ. Ser. I Mat. Mekh. 73 (2005), 55-59.

JSPS Research Fellow, Graduate School of Mathematical Sciences, The University of Tokyo, 3-8-1 Komaba, Meguro, Tokyo, 153-8914 Japan.

E-mail address: yamashu@ms.u-tokyo.ac.jp 